\title{
Solving nth-Order Integro-Differential Equations Using the Combined Laplace Transform-Adomian Decomposition Method
}

\author{
Waleed Al-Hayani \\ Department of Mathematics, College of Computer Science and Mathematics, University of Mosul, Mosul, Iraq \\ Email:waleedalhayani@yahoo.es
}

Received February 23, 2013; revised May 1, 2013; accepted May 8, 2013

Copyright (C) 2013 Waleed Al-Hayani. This is an open access article distributed under the Creative Commons Attribution License, which permits unrestricted use, distribution, and reproduction in any medium, provided the original work is properly cited.

\begin{abstract}
In this paper, the Combined Laplace Transform-Adomian Decomposition Method is used to solve $n$ th-order integro-differential equations. The results show that the method is very simple and effective.
\end{abstract}

Keywords: Integro-Differential Equations; Laplace Transform Method; Adomian Decomposition Method

\section{Introduction}

In the recent literature there is a growing interest to solve integro-differential equations. The reader is referred to [1-3] for an overview of the recent work in this area. In the beginning of the 1980's, Adomian [4-7] proposed a new and fruitful method (so-called the Adomian decomposition method) for solving linear and nonlinear (algebraic, differential, partial differential, integral, etc.) equations. It has been shown that this method yields a rapid convergence of the solutions series to linear and nonlinear deterministic and stochastic equations. The main objective of this work is to use the Combined Laplace Transform-Adomian Decomposition Method (CLT-ADM) in solving the $n$ th-order integro-differential equations.

Let us consider the general functional equation

$$
y-N y=f,
$$

where $N$ is a nonlinear operator, $f$ is a known function, and we are seeking the solution $y$ satisfying (1.1). We assume that for every $f$, Equation (1.1) has one and only one solution.

The Adomian's technique consists of approximating the solution of (1.1) as an infinite series

$$
y=\sum_{n=0}^{\infty} y_{n},
$$

and decomposing the nonlinear operator $N$ as

$$
N y=\sum_{n=0}^{\infty} A_{n},
$$

where $A_{n}$ are polynomials (called Adomian polynomials) of $y_{0}, y_{1}, \cdots, y_{n}$ [4-7] given by

$$
A_{n}=\frac{1}{n !} \frac{\mathrm{d}^{n}}{\mathrm{~d} \lambda^{n}}\left[N\left(\sum_{n=0}^{\infty} \lambda^{i} y_{i}\right)\right]_{\lambda=0}, n=0,1,2, \cdots .
$$

The proofs of the convergence of the series $\sum_{n=0}^{\infty} y_{n}$ and $\sum_{n=0}^{\infty} A_{n}$ are given in [6,8-12]. Substituting (1.2) and (1.3) into (1.1) yields

$$
\sum_{n=0}^{\infty} y_{n}-\sum_{n=0}^{\infty} A_{n}=f .
$$

Thus, we can identify

$$
\begin{aligned}
& y_{0}=f, \\
& y_{n+1}=A_{n}\left(y_{0}, y_{1}, \cdots, y_{n}\right), n=0,1,2, \cdots .
\end{aligned}
$$

Thus all components of $y$ can be calculated once the $A_{n}$ are given. We then define the $n$-terms approximant to the solution $y$ by $\varnothing_{n}[y]=\sum_{i=0}^{n-1} y_{i}$ with $\lim _{n \rightarrow \infty} \varnothing_{n}[y]=y$.

\section{General $n$ th-Order Integro-Differential Equations}

Let us consider the general $n t h$-order integro-differential equations of the type $[1,2]$ : 


$$
\begin{aligned}
& y^{(n)}(x)+f(x) y(x)+\int_{a}^{b} k(x, t) y^{(m)}(t) \mathrm{d} t=g(x), \\
& a<x<b
\end{aligned}
$$

with initial conditions

$$
y(a)=\alpha_{0}, y^{\prime}(a)=\alpha_{1}, y^{\prime \prime}(a)=\alpha_{2}, \cdots, y^{(n-1)}(a)=\alpha_{n-1},
$$

where $\alpha_{i}, i=0,1, \cdots, n-1$ are real constants, $m$ and $n$ are integers and $m<n$. In Equation (2.1) the functions $f(x), g(x)$ and the kernel $k(x, t)$ are given real-valued functions, and $y(x)$ is the solution to be determined. We assume that Equation (2.1) has the unique solution.

To solve the general $n$ th-order integro-differential Equation (2.1) using, the Laplace transform method, we recall that the Laplace transforms of the derivatives of $y(x)$ are defined by

$$
\begin{aligned}
\mathcal{L}\left\{y^{(n)}(x)\right\}(s)= & s^{n} \mathcal{L}\{y(x)\}(s)-s^{n-1} y(0) \\
& -s^{n-2} y^{(0)}-\cdots-y^{(n-1)}(0) .
\end{aligned}
$$

Applying the Laplace transform $\mathcal{L}$ to both sides of (2.1) and taking into account the fact that the convolution theorem for Laplace transform $[13,14]$ gives:

$$
\begin{aligned}
& s^{n} \mathcal{L}\{y(x)\}(s)-s^{n-1} y(0)-s^{n-2} y^{\prime}(0)-\cdots-y^{(n-1)}(0) \\
= & \mathcal{L}\{g(x)\}(s)-\mathcal{L}\{f(x) * y(x)\}(s) \\
& -\mathcal{L}\left\{\int_{a}^{b} k(x, t) y^{(m)}(t) \mathrm{d} t\right\}(s) \\
= & \mathcal{L}\{g(x)\}(s)-\mathcal{L}\{f(x)\}(s) \mathcal{L}\{y(x)\}(s) \\
& -\int_{a}^{b} \mathcal{L}\{k(x, t)\}(s) y^{(m)}(t) \mathrm{d} t .
\end{aligned}
$$

This can be reduced to

$$
\begin{aligned}
\mathcal{L}\{y(x)\}(s) \\
=\frac{s^{n-1} y(0)+s^{n-2} y^{\prime}(0)+\cdots+y^{(n-1)}(0)}{s^{n}+\mathcal{L}\{f(x)\}(s)}+\frac{\mathcal{L}\{g(x)\}(s)}{s^{n}+\mathcal{L}\{f(x)\}(s)} \\
-\frac{1}{s^{n}+\mathcal{L}\{f(x)\}(s)} \int_{a}^{b} \mathcal{L}\{k(x, t)\}(s) y^{(m)}(t) \mathrm{d} t .
\end{aligned}
$$

Substituting (1.2) into (2.2) leads to

$$
\begin{aligned}
\mathcal{L}\left\{\sum_{n=0}^{\infty} y_{n}(x)\right\}(s) \\
=\frac{s^{n-1} y(0)+s^{n-2} y^{\prime}(0)+\cdots+y^{(n-1)}(0)}{s^{n}+\mathcal{L}\{f(x)\}(s)}+\frac{\mathcal{L}\{g(x)\}(s)}{s^{n}+\mathcal{L}\{f(x)\}(s)} \\
-\frac{1}{s^{n}+\mathcal{L}\{f(x)\}(s)} \int_{a}^{b} \mathcal{L}\{k(x, t)\}(s) \sum_{n=0}^{\infty} y_{n}^{(m)}(t) \mathrm{d} t .
\end{aligned}
$$

The Adomian decomposition method presents the recursive relation

$$
\begin{aligned}
& \mathcal{L}\left\{y_{0}(x)\right\}(s) \\
= & \frac{s^{n-1} y(0)+s^{n-2} y^{\prime}(0)+\cdots+y^{(n-1)}(0)}{s^{n}+\mathcal{L}\{f(x)\}(s)}+\frac{\mathcal{L}\{g(x)\}(s)}{s^{n}+\mathcal{L}\{f(x)\}(s)} \\
& \mathcal{L}\left\{y_{n+1}(x)\right\}(s) \\
= & -\frac{1}{s^{n}+\mathcal{L}\{f(x)\}(s)} \int_{a}^{b} \mathcal{L}\{k(x, t)\}(s) y_{n}^{(m)}(t) \mathrm{d} t, \\
n= & 0,1,2, \cdots .
\end{aligned}
$$

A necessary condition for (2.3) to comply is that

$$
\lim _{s \rightarrow \infty} \frac{1}{s^{n}+\mathcal{L}\{f(x)\}(s)}=0 .
$$

Applying the inverse Laplace transform to both sides of the first part of (2.3) gives $y_{0}(x)$, and using the recursive relation (2.3) gives the components of $y_{n}(x), n \geq 0$. We then define the $n$-terms approximant to the solution $y(x)$ by $\phi_{n}[y(x)]=\sum_{i=0}^{n-1} y_{i}(x)$ with

$\lim _{n \rightarrow \infty} \phi_{n}[y(x)]=y(x)$. In this paper, the obtained series solution converges to the exact solution.

\subsection{A Test of Convergence}

The convergence of the method is established by Theorem 3.1 in [9]. In fact, on each interval the inequality $\left\|y_{i+1}\right\|_{2}<\alpha\left\|y_{i}\right\|_{2}$ is required to hold for $i=0,1, \cdots, n$, where $0<\alpha<1$ is a constant and $n$ is the maximum order of the approximant used in the computation. Of course, this is only a necessary condition for convergence, because it would be necessary to compute $\left\|y_{i}\right\|_{2}$ for every $i=0,1, \cdots, n$ in order to conclude that the series is convergent.

\subsection{Definition}

Let $\phi_{n}(x), n=1,2, \cdots$ be the successive approximations to the solution $y(x)$ of a problem. If the positive constants $K, p$ exist such that

$$
K=\lim _{n \rightarrow \infty} \frac{\left|\phi_{n+1}\left(x_{i}\right)-y\left(x_{i}\right)\right|}{\left|\phi_{n}\left(x_{i}\right)-y\left(x_{i}\right)\right|^{p}},
$$

then we call $p$ the (estimated) Local Order of Convergence (EOC) at the point $x_{i}$. The constant $K$ is called Convergence Factor at $x_{i}$.

\section{Applications}

In this section, the CLT-ADM for solving nth-order inte- 
gro-differential equations is illustrated in the three examples given below. To show the high accuracy of the solution results from applying the present method to our problem (2.1) compared with the exact solution, the maximum error is defined as:

$$
E_{n}=\left\|y_{\text {Exact }}(x)-\phi_{n}(x)\right\|_{\infty},
$$

where $n=1,2, \cdots$ represents the number of iterations. Moreover, we give a comparison among the CLT-ADM, Homotopy perturbation method (HPM) [1] and the variational iteration method (VIM) [2]. The computations associated with the examples were performed using Maple 13 package.

\section{Example 1}

Solve the second-order integro-differential equation by using the CLT-ADM [1,2]:

$$
\left\{\begin{array}{l}
y^{\prime \prime}(x)=\mathrm{e}^{x}-x+\int_{0}^{1} x t y(t) \mathrm{d} t, \\
y(0)=1, y^{\prime}(0)=1
\end{array}\right.
$$

As mentioned above, taking Laplace transform of both sides of (3.1) gives

$$
\mathcal{L}\left\{y^{\prime \prime}(x)\right\}(s)=\mathcal{L}\left\{\mathrm{e}^{x}-x\right\}(s)+\mathcal{L}\left\{\int_{0}^{1} x t y(t) \mathrm{d} t\right\}(s)
$$

so that

$$
s^{2} Y(s)-s y(0)-y^{\prime}(0)=\frac{1}{s-1}-\frac{1}{s^{2}}+\frac{1}{s^{2}} \int_{0}^{1} t y(t) \mathrm{d} t
$$

or equivalently

$$
Y(s)=\frac{1}{s}+\frac{1}{s^{2}}-\frac{1}{s^{4}}+\frac{1}{s^{2}(s-1)}+\frac{1}{s^{4}} \int_{0}^{1} t y(t) \mathrm{d} t
$$

where $\mathcal{L}\{y(x)\}(s)=Y(s)$. Substituting the series assumption for $Y(s)$ as given above in (1.2), and using the recursive relation (2.3) we obtain

$$
\begin{aligned}
& Y_{0}(s)=\frac{1}{s}+\frac{1}{s^{2}}-\frac{1}{s^{4}}+\frac{1}{s^{2}(s-1)}, \\
& \mathcal{L}\left\{y_{n+1}(x)\right\}(s)=\frac{1}{s^{4}} \int_{0}^{1} t y_{n}(t) \mathrm{d} t, n=0,1,2, \cdots .
\end{aligned}
$$

Taking the inverse Laplace transform of both sides of the first part of (3.2) gives $y_{0}(x)$, and using the recursive relation (3.2) gives

$$
\begin{gathered}
y_{0}(x)=\mathrm{e}^{x}-\frac{1}{3 !} x^{3}, \\
\vdots \\
y_{n}(x)=\frac{29}{3 ! \cdot 30^{n}} x^{3}, n=1,2, \cdots .
\end{gathered}
$$

Thus the series solution is given by

$$
\begin{aligned}
& \phi_{n}(x)=\sum_{i=0}^{n-1} y_{i}(x)=\mathrm{e}^{x}-\frac{1}{3 ! \cdot 30^{n-1}} x^{3}, n=1,2, \cdots \\
& y(x)=\lim _{n \rightarrow \infty} \phi_{n}(x)=\lim _{n \rightarrow \infty}\left(\mathrm{e}^{x}-\frac{1}{3 ! \cdot 30^{n-1}} x^{3}\right)=\mathrm{e}^{x}
\end{aligned}
$$

that converges to the exact solution $y_{\text {Exact }}(x)=\mathrm{e}^{x}$. In Table 1, the maximum errors and the EOC are presented for $x=0.2(0.2) 1$. Comparing it with the HPM and VIM results given in [1,2], we notice that the result obtained by the present method is very superior (lower error combined with less number of iterations) to that obtained by HPM and VIM. From Table 1, it can be deduced that, the error decreased monotically with the increment of the integer $n$.

Example 2

Solve the third-order integro-differential equation by using the CLT-ADM [1,2]:

$$
\left\{\begin{array}{l}
y^{\prime \prime \prime}(x)=\sin x-x-\int_{0}^{\pi / 2} x t y^{\prime}(t) \mathrm{d} t \\
y(0)=1, y^{\prime}(0)=0, y^{\prime \prime}(0)=-1
\end{array}\right.
$$

As early mentioned, taking Laplace transform of both sides of (3.3) gives

$$
\mathcal{L}\left\{y^{\prime \prime \prime}(x)\right\}(s)=\mathcal{L}\{\sin x-x\}(s)-\mathcal{L}\left\{\int_{0}^{\pi / 2} x t y^{\prime}(t) \mathrm{d} t\right\}(s)
$$

so that

$$
\begin{aligned}
& s^{3} Y(s)-s^{2} y(0)-s y^{\prime}(0)-y^{\prime \prime}(0) \\
= & \frac{1}{s^{2}+1}-\frac{1}{s^{2}}-\frac{1}{s^{2}} \int_{0}^{\pi / 2} t y^{\prime}(t) \mathrm{d} t
\end{aligned}
$$

or equivalently

$$
Y(s)=\frac{1}{s}-\frac{1}{s^{3}}-\frac{1}{s^{5}}+\frac{1}{s^{3}\left(s^{2}+1\right)}-\frac{1}{s^{5}} \int_{0}^{\pi / 2} t y^{\prime}(t) \mathrm{d} t
$$

where $\mathcal{L}\{y(x)\}(s)=Y(s)$. Substituting the series assumption for $Y(s)$ as given above in (1.2), and using the recursive relation (2.3) we obtain

$$
\begin{aligned}
& Y_{0}(s)=\frac{1}{s}-\frac{1}{s^{3}}-\frac{1}{s^{5}}+\frac{1}{s^{3}\left(s^{2}+1\right)}, \\
& \mathcal{L}\left\{y_{n+1}(x)\right\}(s)=-\frac{1}{s^{5}} \int_{0}^{\pi / 2} t y_{n}^{\prime}(t) \mathrm{d} t, n=0,1,2, \cdots .
\end{aligned}
$$

Table 1. Maximum error and EOC for Example 1.

\begin{tabular}{ccccc}
\hline$x$ & $E_{3}$ & $E_{6}$ & $E_{8}$ & EOC \\
\hline 0.2 & $1.4815 \mathrm{E}-06$ & $5.4870 \mathrm{E}-11$ & $6.0966 \mathrm{E}-14$ & 0.99999 \\
0.4 & $1.1852 \mathrm{E}-05$ & $4.3896 \mathrm{E}-10$ & $4.8773 \mathrm{E}-13$ & 1.00000 \\
0.6 & $4.0000 \mathrm{E}-05$ & $1.4815 \mathrm{E}-09$ & $1.6461 \mathrm{E}-12$ & 0.99999 \\
0.8 & $9.4815 \mathrm{E}-05$ & $3.5117 \mathrm{E}-09$ & $3.9018 \mathrm{E}-12$ & 1.00000 \\
1.0 & $1.8519 \mathrm{E}-04$ & $6.8587 \mathrm{E}-09$ & $7.6208 \mathrm{E}-12$ & 1.00000 \\
\hline
\end{tabular}

According to the requirements of our test, $\frac{\left\|y_{i+1}\right\|_{2}}{\left\|y_{i}\right\|_{2}}<1$ for all $i=0,1,2, \cdots, n$. 
Taking the inverse Laplace transform of both sides of the first part of (3.4) gives $y_{0}(x)$, and using the recursive relation (3.4) gives

$$
\begin{gathered}
y_{0}(x)=\cos x-\frac{1}{4 !} x^{4}, \\
\vdots \\
y_{n}(x)=\frac{\left(\pi^{5}+960\right) \pi^{5(n-1)}}{4 ! \cdot 960^{n}} x^{4}, n=1,2, \cdots .
\end{gathered}
$$

The series solution is therefore given by

$$
\begin{aligned}
& \phi_{n}(x)=\sum_{i=0}^{n-1} y_{i}(x)=\cos x+\frac{(-1)^{n} \pi^{5(n-1)}}{4 ! \cdot 960^{n-1}} x^{4}, n=1,2, \cdots \\
& y(x)=\lim _{n \rightarrow \infty} \phi_{n}(x)=\lim _{n \rightarrow \infty}\left(\cos x+\frac{(-1)^{n} \pi^{5(n-1)}}{4 ! \cdot 960^{n-1}}\right)=\cos x
\end{aligned}
$$

that converges to the exact solution $y_{\text {Exact }}(x)=\cos x$. In Table 2, the maximum errors and the EOC are shown for $x=0.2(0.2) 1$. Comparing it with the HPM and VIM results given in $[1,2]$, we notice that the result obtained by the present method is very superior (lower error combined with less number of iterations) to that obtained by HPM and VIM. From Table 2, it can be concluded that, the error decreased monotically with the increment of the integer $n$.

\section{Example 3}

Solve the eighth-order integro-differential equation by using the CLT-ADM $[1,2]$ :

$$
\left\{\begin{array}{l}
y^{(8)}(x)=-8 \mathrm{e}^{x}+x^{2}+y(x)+\int_{0}^{1} x^{2} y^{\prime}(t) \mathrm{d} t \\
y(0)=1, y^{\prime}(0)=0, y^{\prime \prime}(0)=-1, y^{\prime \prime \prime}(0)=-2, \\
y^{(4)}(0)=-3, y^{(5)}(0)=-4, y^{(6)}(0)=-5, y^{(7)}(0)=-6 .
\end{array}\right.
$$

As previously mentioned, taking Laplace transform of both sides of (3.5) gives

$$
\begin{aligned}
\mathcal{L}\left\{y^{(8)}(x)\right\}(s)= & \mathcal{L}\left\{-8 \mathrm{e}^{x}+x^{2}+y(x)\right\}(s) \\
& +\mathcal{L}\left\{\int_{0}^{1} x^{2} y^{\prime}(t) \mathrm{d} t\right\}(s)
\end{aligned}
$$

Table 2. Maximum error and EOC for Example 2.

\begin{tabular}{ccccc}
\hline$x$ & $E_{3}$ & $E_{6}$ & $E_{8}$ & $E O C$ \\
\hline 0.2 & $6.7743 \mathrm{E}-06$ & $2.1943 \mathrm{E}-07$ & $2.2297 \mathrm{E}-08$ & 0.99999 \\
0.4 & $1.0839 \mathrm{E}-04$ & $3.5109 \mathrm{E}-06$ & $3.5676 \mathrm{E}-07$ & 0.99999 \\
0.6 & $5.4872 \mathrm{E}-04$ & $1.7774 \mathrm{E}-05$ & $1.8061 \mathrm{E}-06$ & 1.00000 \\
0.8 & $1.7342 \mathrm{E}-03$ & $5.6175 \mathrm{E}-05$ & $5.7082 \mathrm{E}-06$ & 1.00000 \\
1.0 & $4.2339 \mathrm{E}-03$ & $1.3714 \mathrm{E}-04$ & $1.3936 \mathrm{E}-05$ & 0.99999 \\
\hline
\end{tabular}

According to the requirements of our test, $\frac{\left\|y_{i+1}\right\|_{2}}{\left\|y_{i}\right\|_{2}}<1$ for all $i=0,1,2, \cdots, n$. so that

$$
\begin{aligned}
& s^{8} Y(s)-s^{7} y(0)-s^{6} y^{\prime}(0)-s^{5} y^{\prime \prime}(0)-s^{4} y^{\prime \prime \prime}(0) \\
& -s^{3} y^{(4)}(0)-s^{2} y^{(5)}(0)-s y^{(6)}(0)-y^{(7)}(0) \\
& =\frac{-8}{s-1}+\frac{2}{s^{3}}+Y(s)+\frac{2}{s^{3}} \int_{0}^{1} y^{\prime}(t) \mathrm{d} t
\end{aligned}
$$

or equivalently

$$
\begin{aligned}
& Y(s) \\
= & \frac{s^{7}}{s^{8}-1}-\frac{s^{5}}{s^{8}-1}-\frac{2 s^{4}}{s^{8}-1}-\frac{3 s^{3}}{s^{8}-1}-\frac{4 s^{2}}{s^{8}-1}-\frac{5 s}{s^{8}-1}-\frac{6}{s^{8}-1} \\
& +\frac{2}{s^{3}\left(s^{8}-1\right)}-\frac{8}{(s-1)\left(s^{8}-1\right)}+\frac{2}{s^{3}\left(s^{8}-1\right)} \int_{0}^{1} y^{\prime}(t) \mathrm{d} t
\end{aligned}
$$

where $\mathcal{L}\{y(x)\}(s)=Y(s)$. Substituting the series assumption for $Y(s)$ as given above in (1.2), and using the recursive relation (2.3) we obtain

$$
\begin{aligned}
Y_{0}(s)= & \frac{s^{7}}{s^{8}-1}-\frac{s^{5}}{s^{8}-1}-\frac{2 s^{4}}{s^{8}-1}-\frac{3 s^{3}}{s^{8}-1}-\frac{4 s^{2}}{s^{8}-1}-\frac{5 s}{s^{8}-1} \\
& -\frac{6}{s^{8}-1}+\frac{2}{s^{3}\left(s^{8}-1\right)}-\frac{8}{(s-1)\left(s^{8}-1\right)} \\
& \mathcal{L}\left\{y_{n+1}(x)\right\}(s)=\frac{2}{s^{3}\left(s^{8}-1\right)} \int_{0}^{1} y_{n}^{\prime}(t) \mathrm{d} t, n=0,1,2, \cdots .
\end{aligned}
$$

Taking the inverse Laplace transform of both sides of the first part of (3.6) gives $y_{0}(x)$, and using the recursive relation (3.6) gives

$$
\begin{aligned}
& y_{0}(x)=\frac{5}{4} \mathrm{e}^{x}+\frac{1}{4} \mathrm{e}^{-x}-x \mathrm{e}^{x}-x^{2}-\frac{1}{2} \cos x \\
& +\sin \left(\frac{\sqrt{2}}{2} x\right) \sinh \left(\frac{\sqrt{2}}{2} x\right), \\
& y_{1}(x)=x^{2}+\frac{1}{2} \cos x-\frac{1}{2} \cosh x+\sin \left(\frac{\sqrt{2}}{2} x\right) \sinh \left(\frac{\sqrt{2}}{2} x\right), \\
& y_{2}(x)=0.5512 \times 10^{-6} x^{2}+0.2756 \times 10^{-6} \cos x-0.2756 \\
& \times 10^{-6} \cosh x-0.5512 \times 10^{-6} \sin \left(\frac{\sqrt{2}}{2} x\right) \sinh \left(\frac{\sqrt{2}}{2} x\right), \\
& y_{3}(x)=0.3038 \times 10^{-12} x^{2}+0.1519 \times 10^{-12} \cos x-0.1519 \\
& \times 10^{-12} \cosh x-0.3038 \times 10^{-12} \sin \left(\frac{\sqrt{2}}{2} x\right) \sinh \left(\frac{\sqrt{2}}{2} x\right), \\
& y_{4}(x)=0.1674 \times 10^{-18} x^{2}+0.8371 \times 10^{-19} \cos x-0.8371 \\
& \times 10^{-19} \cosh x-0.1674 \times 10^{-18} \sin \left(\frac{\sqrt{2}}{2} x\right) \sinh \left(\frac{\sqrt{2}}{2} x\right),
\end{aligned}
$$

and so on for other components. Consequently, the series solution is given by 
Table 3. Maximum error and EOC for Example 3.

\begin{tabular}{cccc}
\hline$x$ & $E_{1}$ & $E_{2}$ & $E_{3}$ \\
\hline 0.2 & $5.6437 \mathrm{E}-14$ & $0.3111 \mathrm{E}-19$ & 0.99999 \\
0.4 & $5.7792 \mathrm{E}-11$ & $0.3185 \mathrm{E}-16$ & 0.99999 \\
0.6 & $3.3326 \mathrm{E}-09$ & $0.1837 \mathrm{E}-14$ & 0.99999 \\
0.8 & $5.9179 \mathrm{E}-08$ & $0.3262 \mathrm{E}-13$ & 0.99999 \\
1.0 & $5.5115 \mathrm{E}-07$ & $0.3038 \mathrm{E}-12$ & 1.00000 \\
\hline
\end{tabular}

According to the requirements of our test, $\frac{\left\|y_{i+1}\right\|_{2}}{\left\|y_{i}\right\|_{2}}<1$ for all $i=0,1,2, \cdots, n$.

$$
\begin{aligned}
& y(x)=\lim _{n \rightarrow \infty} \phi_{n}(x) \\
& =\lim _{n \rightarrow \infty} \sum_{i=0}^{n-1} y_{i}(x)=\frac{5}{4} \mathrm{e}^{x}+\frac{1}{4} \mathrm{e}^{-x}-x \mathrm{e}^{x}-\frac{1}{2} \cosh x=(1-x) \mathrm{e}^{x}
\end{aligned}
$$

that converges to the exact solution $y_{\text {Exact }}(x)=(1-x) \mathrm{e}^{x}$. In Table 3, the maximum errors and the EOC are given for $x=0.2(0.2) 1$. Comparing it with the VIM results given in [2], we realize that the result obtained by the present method is very superior (lower error combined with less number of iterations) to that obtained by VIM. From Table 3, it can be deduced that, the error decreased monotically with the increment of the integer $n$.

\section{Conclusion}

The CLT-ADM has been applied for solving nth-order integro-differential equations. Comparison of the results obtained by the present method with that obtained by HPM and VIM reveals that the present method is superior because of the lower error and less number of needed iteration. It has been shown that error is monotically reduced with the increment of the integer $n$.

\section{Acknowledgements}

We would like to thank the referees for their careful review of our manuscript.

\section{REFERENCES}

[1] A. Golbabai and M. Javidi, "Application of He's Homotopy Perturbation Method for nth-Order Integro-Differential Equations," Applied Mathematics and Computation, Vol. 190, No. 2, 2007, pp. 1409-1416. doi:10.1016/i.amc.2007.02.018
[2] X. F. Shang and D. F. Han, "Application of the Variational Iteration Method for Solving nth-Order IntegroDifferential Equations," Journal of Computational and Applied Mathematics, Vol. 234, No. 5, 2010, pp. 14421447. doi:10.1016/i.cam.2010.02.020

[3] A. M. Wazwaz, "The-Combined Laplace Transform-Adomian Decomposition Method for Handling Nonlinear Volterra-Integro Differential Equations," Applied Mathematics and Computation, Vol. 216, No. 4, 2010, pp. 1304-1309. doi:10.1016/j.amc.2010.02.023

[4] G. Adomian, "Stochastic Systems," Academic Press, New York, 1983.

[5] G. Adomian, "Nonlinear Stochastic Operator Equations," Academic Press, New York, 1986.

[6] G. Adomian, "Nonlinear Stochastic Systems Theory and Applications to Physics," Kluwer Academic Publishers, Dordrecht, 1989. doi:10.1007/978-94-009-2569-4

[7] G. Adomian, "Solving Frontier Problems of Physics: The Decomposition Method," Kluwer Academic Publishers, Dordrecht, 1994.

[8] K. Abbaoui and Y. Cherruault, "Convergence of Adomian's Method Applied to Differential Equations," Mathematical and Computer Modelling, Vol. 28, No. 5, 1994, pp. 103-109.

[9] K. Abbaoui and Y. Cherruault, "New Ideas for Proving Convergence of Decomposition Methods," Computers and Mathematics with Applications, Vol. 29, No. 7, 1995, pp. 103-108. doi:10.1016/0898-1221(95)00022-Q

[10] K. Abbaoui and Y. Cherruault, "Convergence of Adomian's Method Applied to Nonlinear Equations," Mathematical and Computer Modelling, Vol. 20, No. 9, 1994, pp. 60-73. doi:10.1016/0895-7177(94)00163-4

[11] Y. Cherruault and G. Adomian, "Decomposition Methods: a New Proof of Convergence," Mathematical and Computer Modelling, Vol. 18, No. 12, 1993, pp. 103-106. doi:10.1016/0895-7177(93)90233-O

[12] S. Guellal and Y. Cherruault, "Practical Formula for Calculation of Adomian's Polynomials and Application to the Convergence of the Decomposition Method," International Journal Bio-Medical Computing, Vol. 36, No. 3, 1994, pp. 223-228. doi:10.1016/0020-7101(94)90057-4

[13] A. D. Polyanin and A. V. Manzhirov, "Handbook of Integral Equations," CRC Press, New York, 1998. doi:10.1201/9781420050066

[14] A. J. Jerri, "Introduction to Integral Equations with Applications," Wiley, New York, 1999. 